\title{
Multimodal Treatment of cT3 Rectal Cancer in a Prospective Multi-Center Observational Study: Can Neoadjuvant Chemoradiation Be Omitted in Patients with an MRI-Assessed, Negative Circumferential Resection Margin?
}

\author{
Henry Ptok ${ }^{a, b}$ Frank Meyer ${ }^{a, b}$ Ingo Gastinger ${ }^{b}$ Benjamin Garlipp ${ }^{c}$ \\ a Department of General, Abdominal, Vascular and Transplant Surgery, University Hospital Magdeburg, \\ Magdeburg, Germany; ${ }^{b}$ Institute of Quality Assurance in Operative Medicine, University of Magdeburg, \\ Magdeburg, Germany; ${ }^{C}$ Department of Surgery (Oranienburg), Regional Hospital (Oberhavel Kliniken) \\ at Hennigsdorf, Hennigsdorf, Germany
}

\section{Keywords}

Rectal cancer · Neoadjuvant chemoradiotherapy ·

Circumferential resection margin $\cdot$ Outcome $\cdot$ Multi-center prospective observational study

\begin{abstract}
Background/Aim: Neoadjuvant chemoradiation (nCRT) in rectal cancer is associated with significant long-term morbidity. It is unclear whether $\mathrm{nCRT}$ in resectable mesorectal fascia circumferential resection margin ( $\mathrm{mrCRM}$ )-negative rectal cancer treated by adequate total mesorectal excision (TME) is beneficial. The aim was to determine if $\mathrm{nCRT}$ can be omitted in patients with MRI-assessed cT3 rectal cancer and a negative mrCRM undergoing good-quality TME. Methods: By means of a prospective nationwide registry $(n=43.147$; prospective multi-center observational study), patients with CT3 rectal cancer $<12 \mathrm{~cm}$ from the anal verge with a negative (>1 mm) MRI-assessed CRM undergoing radical resection from 2006 to 2008 were selected. Overall, 87 patients were available for the final analysis (TME-alone, $n=25 ; \mathrm{nCRT}+\mathrm{TME}$, $n=62$ ). Groups were balanced for age, sex, and ASA score, with a nonsignificant predominance of males in the nCRT+TME group. As main outcome measures, local and distant recurrence rates were compared between patients undergoing primary surgery (TME-alone) vs. neoadjuvant chemoradiation + surgery (nCRT+TME). Results: In the TME-
\end{abstract}

karger@karger.com www.karger.com/vis

Karger" 马OPEN ACCESS
(C) 2021 The Author(s)

Published by S. Karger AG, Basel

This is an Open Access article licensed under the Creative Common Attribution-NonCommercial-4.0 International License (CC BY-NC) (http://www.karger.com/Services/OpenAccessLicense), applicable to the online version of the article only. Usage and distribution for commercial purposes requires written permission. alone group, tumors were located closer to the anal verge $(p=0.018)$ and demonstrated a smaller minimal circumferential distance from the resection margin $(p=0.036)$. TME quality was comparable, as was median follow-up (48.9 vs. 44.9 months; $p=0.268$ ). Local recurrences occurred at a similar rate in the TME-alone $(n=1 ; 5.3 \%)$ and $\mathrm{nCRT}+\mathrm{TME}$ groups $(n=3 ; 5.5 \%)(p=0.994)$ and were diagnosed at 10 months (TME-alone) and at 8, 13, and 18 months (nCRT+TME). Distant recurrences occurred in 28.9 and $17.4 \%$ of the cases, respectively $(p=0.626)$. The analysis was limited to cT3 cancers with a negative $\mathrm{mrCRM}$. In addition, caution is required when appraising these results because of the limited number of evaluable subjects (especially in the TME-alone group), which adds some uncertainty to the statistical analysis. Conclusions: In this cohort of patients with rectal cancer located $<12 \mathrm{~cm}$ from the anal verge and a negative mrCRM undergoing adequate TME, omission of $\mathrm{nCRT}$ had no impact onto the local recurrence rate.

(C) 2021 The Author(s) Published by S. Karger AG, Basel

\section{Background}

Until the early 1990s, rectal cancer resections were associated with a 5-year local recurrence rate between 20 and $45 \%$ [1-3]. This was the rationale for investigating a multimodal treatment regimen including preoperative 
$5 \times 5$ Gy short-course radiation in Sweden in the 1980s, with the aim being to improve local tumor control [4-7]. Local recurrence rates were reduced by $50 \%$ but still remained high (around $15-20 \%$ at 5 years). It was not until the adoption of total mesorectal excision (TME) as described by Heald et al. [8] that 5-year local recurrence rates of $8.2 \%$ were achieved in the Swedish TME project [9].

In a similar effort to improve local tumor control, preoperative $5 \times 5$ Gy short-course radiation was investigated in the randomized-controlled Dutch TME trial in the 1990s [10]. In that study, surgery was performed according to the TME principles after dedicated training of the participating surgeons. As a result, local recurrences were reduced from 10.9 to $5.6 \%$ at 5 years in the combinedmodality arm compared to the surgery-alone arm; however, the significant benefit from preoperative radiation was limited to UICC stage III rectal cancers and cancers localized in the mid-rectum $[11,12]$. This trial remains the only randomized controlled trial (RCT) investigating the impact of preoperative radiation in resectable rectal cancer treated by standardized TME. Still, comparing these results with the local recurrence rate after TME alone reported by Heald et al. [8] in 1998 (6\% at 5 years) casts some doubt on the benefit of neoadjuvant radiotherapy in resectable rectal cancer treated by optimized surgery.

As with the Swedish TME project [9], the Dutch TME trial was started immediately after the TME surgical principles had been adopted and training of the participating surgeons had been completed, resulting in a relatively large proportion of incomplete TME surgeries and specimens with a positive circumferential resection margin (CRM) in that study $[13,14]$. This may have influenced the recurrence rates and must be taken into account when interpreting these results.

In Germany, neoadjuvant chemoradiation or neoadjuvant short-course radiation became the nationwide treatment standard for resectable UICC stage II and III rectal cancers in the early 2000s [15]. As from 2007/2008, almost $50 \%$ of all rectal cancers $(75 \%$ of all clinical stage III rectal cancers) received preoperative radiation, predominantly as a part of 5-FU-based chemoradiation therapy. The rate of incomplete TME surgeries (MERCURY grade III) was approximately $3 \%$. Local recurrences occur in $4.6 \%$ of all stage I to III patients at 5 years [15].

Considering radiation-associated long-term morbidity [16], as well as the fact that adequate TME surgery is currently performed in most institutions, the current standard of administering neoadjuvant (chemo) radiation therapy to all stage II/III rectal cancer patients with tumor lesions located at $<10 \mathrm{~cm}$ from the anal verge has increasingly been called into question. Recent study results suggest that a local recurrence rate around $5 \%$ can be achieved in cancers located in the lower two thirds of the rectum even without neoadjuvant radiation if pretherapeutic imaging demonstrates a clear CRM [17-19].

In this report based on a prospective multi-center observational study, we compared long-term outcomes in patients with locally advanced rectal cancer located in the lower 2 rectal thirds with a tumor-negative CRM on preoperative magnetic resonance imaging undergoing either primary TME or neoadjuvant radiation and subsequent TME under routine conditions of daily surgical practice.

\section{Methods}

\section{Data Source}

This analysis is based on data from the prospective, multi-center observational study on quality assurance in rectal cancer care performed by the "AN-Institut für Qualitätssicherung in der operativen Medizin” at Otto-von-Guericke University (Magdeburg, Germany). In this registry, demographic data and diagnostic-, tumor-, and treatment-related parameters, as well as histopathology results from rectal cancer patients, are prospectively documented. Follow-up information is obtained annually from the treating institutions as well as from general practitioners. Hospitals throughout Germany can enter their data on a voluntary basis. Written informed consent from patients is required before data collection. Since 2000, $>40,000$ patients undergoing surgery for rectal cancer have been documented.

\section{Study Design/Subjects}

From the registry, all patients undergoing TME for histologically proven, nonmetastatic rectal cancer located $<12 \mathrm{~cm}$ from the anal verge between January 1, 2006, and December 31, 2008, were selected for whom preoperative MR imaging demonstrating cT3 stage and a minimal distance of $>1 \mathrm{~mm}$ from the mesorectal fascia (negative circumferential resection margin; mrCRM-) was available.

Obtaining preoperative pelvic MRI is not mandatory for data entry into the quality assurance registry, and information on whether mrCRM was determined in an individual patient was not obtained during primary data collection. Therefore, all patients in whom preoperative MRI had been performed were identified, and their imaging data were obtained from the treating institutions at the time of the analysis. mrCRM was then determined on the original MR images by a radiologist with adequate experience in interpreting rectal cancer MRI. Only patients with mrCRM-negative cT3 rectal cancers were considered for final analysis. These patients were classified into 2 groups (TME-alone vs. neoadjuvant chemoradiotherapy [nCRT] + TME) according to their treatment. Patients undergoing short-course radiotherapy or intensified chemoradiotherapy (i.e., those receiving drugs other than 5-FU with radiotherapy) were excluded.

Analysis

Patient- and tumor-related parameters, TME quality according to the MERCURY classification as assessed by the pathologist, and long-term outcomes (local and distant recurrence rate, diseasefree survival, and overall survival) were compared between groups (TME-alone vs. nCRT+TME). 


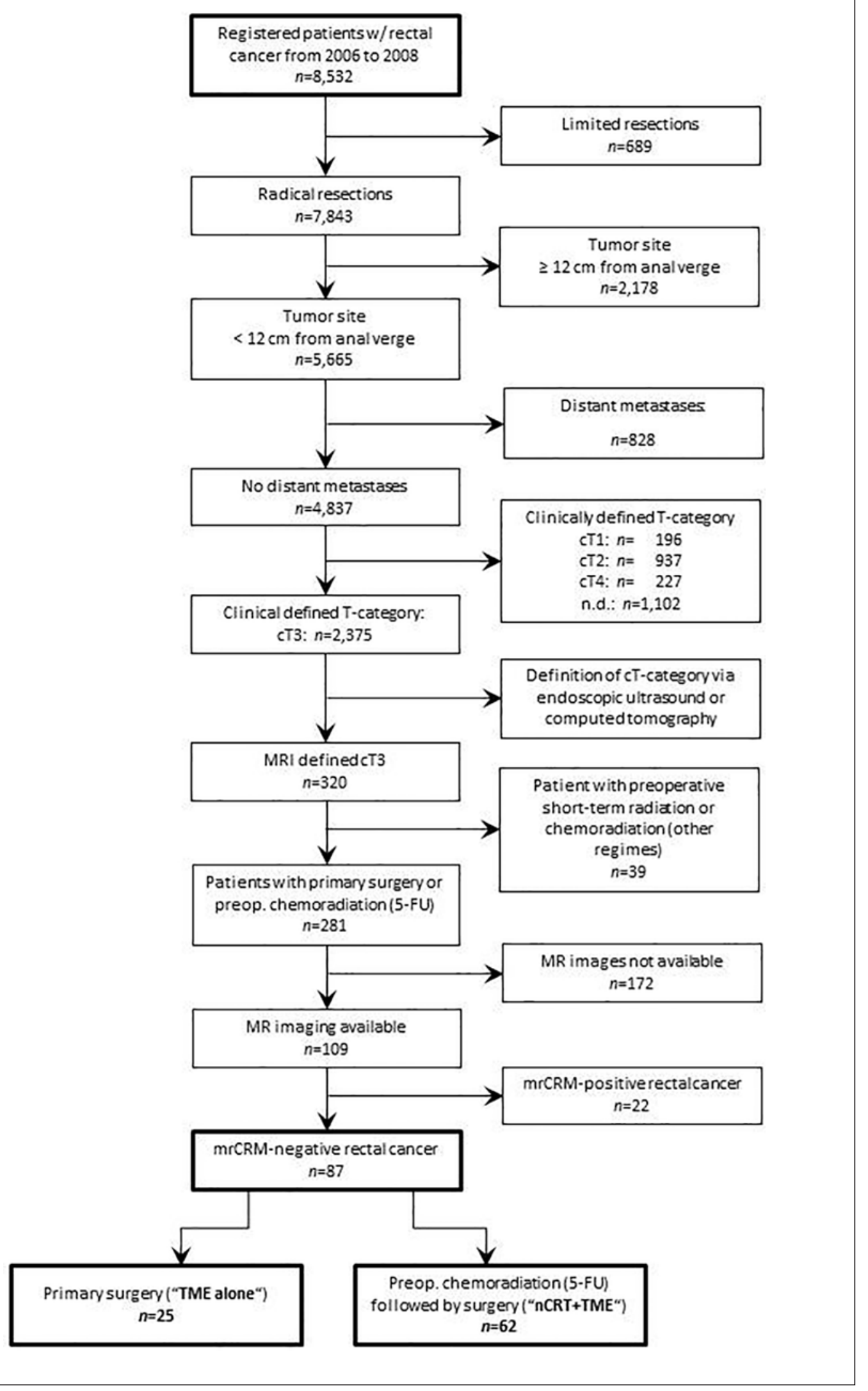

Fig. 1. Patient selection for the current study. n.d., not determined. 
Table 1. Univariate comparison of patient, tumor, and treatment characteristics

\begin{tabular}{|c|c|c|c|}
\hline & $\begin{array}{l}\text { TME-alone group } \\
(n=25)\end{array}$ & $\begin{array}{l}\text { nCRT+TME } \\
\text { group }(n=62)\end{array}$ & $p$ value \\
\hline Age, years & $68.0(62.9-73.1)$ & $66.5(64.0-69.0)$ & 0.545 \\
\hline Male:female ratio & $1.08: 1$ & $2.65: 1$ & 0.081 \\
\hline ASA score & & & 0.883 \\
\hline I & 4.0 & 6.5 & \\
\hline II & 52.0 & 50.0 & \\
\hline III & 40.0 & 41.9 & \\
\hline IV & 4.0 & 1.6 & \\
\hline Tumor location from the anal verge, $\mathrm{cm}$ & & & 0.018 \\
\hline $8-11.9$ & 36.0 & 40.3 & \\
\hline $4-7.9$ & 36.0 & 50.0 & \\
\hline$<4$ & 28.0 & 9.7 & \\
\hline mrCRM, mm & & & 0.036 \\
\hline$>1-2$ & 48.0 & 22.6 & \\
\hline$>2-5$ & 24.0 & 51.6 & \\
\hline$>5-10$ & 28.0 & 21.0 & \\
\hline$>10$ & - & 4.8 & \\
\hline TME quality (MERCURY criteria) & & & 0.445 \\
\hline Grade I & 84.0 & 91.2 & \\
\hline Grade II & 16.0 & 8.8 & \\
\hline Grade III & - & - & \\
\hline (y)pT, \% & & & 0.442 \\
\hline (y)pT1 & 4.0 & 12.9 & \\
\hline (y)pT2 & 32.0 & 25.8 & \\
\hline (y)pT3 & 64.0 & 61.3 & \\
\hline Maximum tumor diameter, $\mathrm{mm}$ & $41.0(35.8-46.2)$ & $21.3(21.2-26.5)$ & $<0.001$ \\
\hline (y)pN positive cases, $\%$ & 40.0 & 37.1 & 0.812 \\
\hline Examined lymph nodes, $n$ & $18.4(15.3-21.5)$ & $14.2(12.8-15.6)$ & 0.017 \\
\hline pCRM, \% & & & 0.495 \\
\hline Negative & 95.5 & 98.4 & \\
\hline Positive & 4.5 & 1.6 & \\
\hline Adjuvant chemotherapy, \% & 36.0 & 87.1 & $<0.001$ \\
\hline Follow-up time, months & $48.9(39.5-58.3)$ & $44.5(40.9-48.0)$ & 0.268 \\
\hline
\end{tabular}

Values are presented as means (95\% CI) or percents unless otehrwise stated. $p$ values in bold indicate significant difference.

\section{Statistics}

SPSS Statistics version 22.0 was used for statistical analysis. Categorical parameters were compared using the $\chi^{2}$ test and the Fisher exact test. Results for categorical variables are reported as absolute and relative frequencies. For continuous parameters, the Student $t$ test was used for comparison between groups. Results are reported as mean values and 95\% CI. Long-term outcomes were analyzed using the Kaplan-Meier method, and univariate comparison between groups was performed using the log-rank test. A 2 -sided $p$ value $<0.05$ was considered statistically significant.

\section{Results}

Of the 8,532 patients entered into the registry during the study period, 2,375 underwent radical resection of nonmetastatic cT3 cancer $<12 \mathrm{~cm}$ from the anal verge. Only 320 of these (13.5\%) underwent preoperative pelvic MRI. MRI scans of 281 potentially suitable patients un- dergoing primary TME or 5-FU-only-based nCRT (Fig. 1) were requested from the treating institutions, of which 109 scans were obtained. Reassessment of these scans revealed a negative mrCRM in 87 patients, who represent the final analysis population. Of these, 25 underwent primary surgery (TME-alone group), whereas 62 underwent nCRT prior to surgery (nCRT+TME group; Fig. 1).

\section{Pre-Therapeutic Patient- and Tumor-Related Parameters}

Age, ASA score, and gender distribution were not significantly different between groups, with a nonsignificant predominance of males in the nCRT+TME group (Table 1). Significant differences were found in tumor location, in tumor lesions of the TME-alone group being located closer to the anal verge than those in the nCRT+TME group ( $p=0.018$ ), as well as in mrCRM. Most patients in 


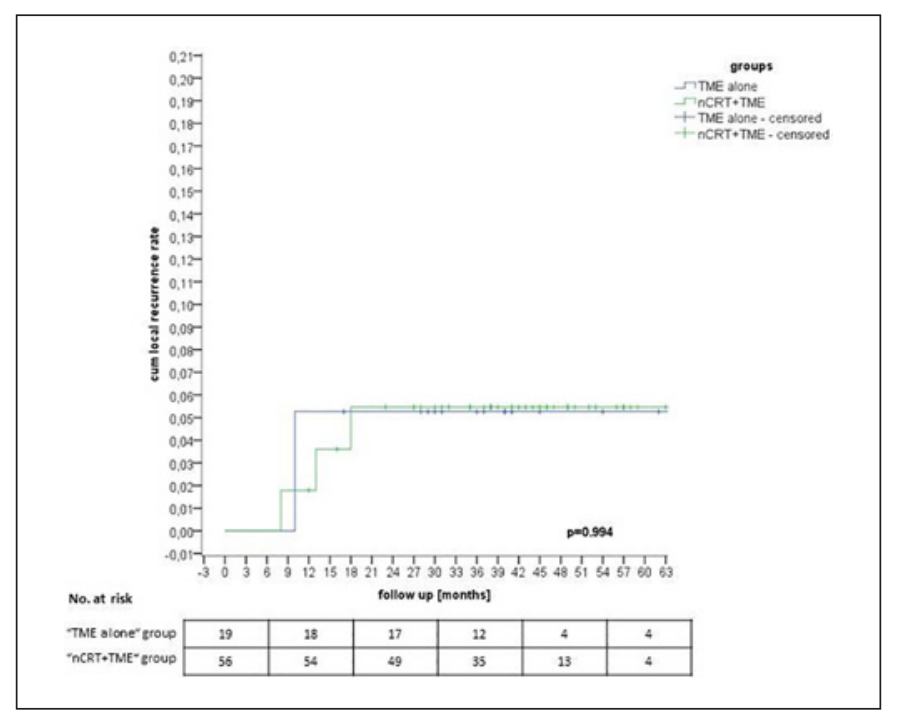

Fig. 2. Cumulative local recurrence rate in the TME-alone and nCRT+TME groups.

the TME-alone group had an mrCRM $>1-2 \mathrm{~mm}$, whereas this was $>2-5 \mathrm{~mm}$ in the majority of patients in the nCRT+TME group $(p=0.036)$.

\section{Postoperative Tumor Characteristics}

TME quality was graded as "good" (grade I) in $84 \%$ of the specimens from the TME-alone group and in $91 \%$ of the specimens from the nCRT+TME group (Table 1). No specimen was assessed as "poor" (grade III) in either group. TME quality was not significantly different between groups $(p=0.445)$.

Postoperative tumor infiltration depth (pT or ypT stage, respectively) was not significantly different between groups $(p=0.442)$, even though patients in the nCRT+TME group showed a tendency toward a lower infiltration depth and the maximum tumor diameter was significantly lower in the nCRT+TME group compared to the TME-alone group $(p<0.001)$.

The mean number of lymph nodes investigated per specimen was significantly lower in the nCRT+TME group compared to the TME-alone group (14.2 vs. 18.4; $p=0.017$ ); however, no difference regarding lymph node positivity (detectable tumor infiltration) between groups was found (TME-alone group, 40.0\%; nCRT+TME group, $37.1 \% ; p=0.812)$. One patient in each group demonstrated a pCRM of $1 \mathrm{~mm}$ or less ( 4.5 vs. $1.6 \% ; p=0.495$ ).

\section{Long-Term Outcomes}

Twenty-two patients in the TME-alone group and 59 patients in the nCRT+TME group consented to having their follow-up information documented in the registry. Follow-up information was available for 19 out of $22 \mathrm{pa}-$ tients in the TME-alone group and for 56 out of 59 pa-

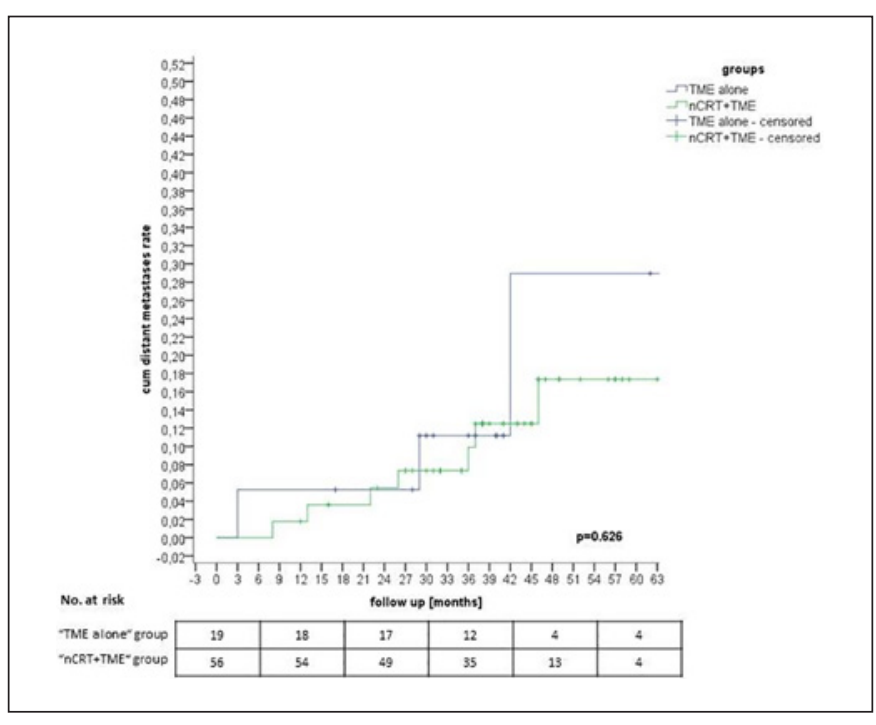

Fig. 3. Cumulative rate of distant recurrences in the TME-alone and nCRT+TME groups.

tients in the nCRT+TME group. The median follow-up was 48.9 vs. 44.5 months in the TME-alone and nCRT+TME groups, respectively $(p=0.268)$.

Kaplan-Meier plots for local recurrence rates are shown in Figure 2. One patient in the TME-alone group developed local recurrence 10 months after surgery. In the nCRT+TME group, local recurrences were detected in 3 patients 8,13 , and 18 months after surgery. The 5 -year local recurrence rate was not different between groups (TME-alone group, 5.3\%; nCRT+TME group, $5.5 \% ; p=0.994)$.

Distant recurrences were diagnosed during follow-up in 3 patients in the TME-alone group and in 8 patients in the nCRT+TME group. The estimated 5-year distant recurrence rate in both groups according to the KaplanMeier method was 28.9 vs. $17.4 \%$, respectively ( $p=0.626$; Fig. 3).

No significant difference in disease-free and overall survival was found between the groups. The 5-year disease-free survival was $71.1 \%$ in the TME-alone group vs. $82.6 \%$ in the nCRT+TME group ( $p=0.626)$. The mean overall survival in both groups was 60 vs. 58.6 months, respectively $(p=0.429)$.

\section{Discussion and Conclusion}

The analysis presented here found a 5.3\% local recurrence rate in patients with MRI-assessed cT3, CRM-negative rectal cancer closer than $12 \mathrm{~cm}$ to the anal verge who underwent upfront TME without neoadjuvant chemoradiation. Comparison of this result with the local recurrence rate of $5.5 \%$ in patients with a similar extent of dis- 
ease treated by nCRT followed by TME indicates that omitting nCRT in these patients does not seem to compromise local tumor control.

The selection of patients for this analysis from the raw data collected within the prospective observational study was intended to generate a cohort of patients with clearly defined disease characteristics (cT3, CRM-, pretherapeutic MRI assessment) suitable to address a clinically relevant issue. A CRM-negative surgical specimen is one of the most relevant factors predicting a low local recurrence rate [20]. Pretherapeutic MRI has been demonstrated to be the most accurate diagnostic modality to predict a negative CRM on subsequent surgery [21, 22]. Hence, pelvic MRI was a prerequisite for inclusion into the current analysis. During the study period, only $28 \%$ of all rectal cancer patients in the registry underwent pretherapeutic MRI [23], which greatly reduced the number of suitable subjects. Moreover, it was necessary to select a study period when the nationwide TME quality in routine care for rectal cancer had achieved an adequate level but neoadjuvant (chemo-) radiation, though recommended by the national guidelines [24], was not yet fully established. Observing all these requirements, only a small number of patients were available for the analysis. Because the incidence of local recurrences was generally low, this constitutes a significant limitation to the validity of any statistical comparison between groups, which must be kept in mind when interpreting our results and precludes any general recommendation to be derived therefrom.

The 5.3\% local recurrence rate with upfront TME presented here is in line with recent results reported by other authors [17-19], who found local recurrence rates between 4.3 and 5.4\%. Kulu et al. [17] retrospectively compared their results with a historical cohort of patients treated by nCRT+TME, whereas Fan et al. [18] performed a prospective, randomized comparison between patients treated with upfront TME or nCRT+TME. Similar to our analysis, local recurrences for primarily resectable rectal cancer did not differ between the cohorts compared in both studies.

Adequate TME quality is a prerequisite for a low local recurrence rate [13, 25]. Heald et al. [8], in 1998, reported local recurrences to occur in $6 \%$ of patients at 5 years after TME who were operated on during a period of almost 20 years. Comparing the first and second halves of their study period, they reported a reduction in local recurrences from 8.5 to $3 \%$ in all patients, or from 4 to $0.5 \%$ in patients undergoing anterior rectal excision with a curative intent. These results clearly demonstrate that a learning curve exists for performing good-quality TME. This must be borne in mind when comparing the data presented here with the results from the Dutch TME trial $[11,12]$ and the MRC CR07/NCIC-CTG C016 trial [26]. Both of these randomized controlled trials included all patients with primarily resectable rectal cancer and were able to demonstrate a significant improvement in local recurrences in patients with low rectal cancer through preoperative short-course radiation. Local recurrence rates were $5.6 \%$ at 5 years [11] and $4.4 \%$ at 3 years [26]. During preparation for the Dutch TME trial, participating surgeons were familiarized with the TME concept through workshops, symposia, and video presentations. Moreover, the first 5 TME procedures in each institution were supervised by an instructor surgeon. Hence, the participating surgeons had limited experience with TME at the time the study was started. In an examination of TME quality in the Dutch TME trial, Nagtegaal et al. [13] found only $57 \%$ of TME specimens to be "complete," whereas $24 \%$ of the TME specimens were classified as "incomplete." In the MRC CR07/NCIC-CTG C016 trial, adherence to the TME technique was merely recommended but no standardized training was required prior to participation in the study. Analyzing TME quality in specimens from this trial, Quirke et al. [25] found 52 and $13 \%$ of them to be excised along the mesorectal and muscularis propria planes of surgery, respectively. In the observational study presented here, TME quality was assessed by the collaborating pathologists at participating centers according to MERCURY criteria. "Good" TME quality was found in 84 and $91 \%$ of specimens in the TME-alone and nCRT+TME groups, respectively. None of the specimens in either group was graded as "poor." The relative improvement in TME quality in our observational study compared to the 2 randomized-controlled trials mentioned may be one factor contributing to the low local recurrence rate achieved with upfront TME in our analysis.

Interestingly, $4.5 \%$ of the specimens in the TME-alone group and $1.6 \%$ of the specimens in the $\mathrm{nCRT}+\mathrm{TME}$ group demonstrated a positive pCRM. pCRM is one of the strongest independent predictive factors for local recurrence following surgery for rectal cancer, and it is dependent on TME quality. As reported by Nagtegaal et al. [13], the rate of pCRM-positive surgical specimens in the Dutch TME trial was 23\%, whereas it was $12 \%$ in the MRC CR07/NCIC-CTG016 trial as reported by Quirke et al. [25]. The substantially lower positive pCRM rate found in the analysis presented here can be considered another contributing factor for the reduced rate of local recurrences observed after TME alone. In the study by Kulu et al. [17], an even lower positive pCRM rate $(1.8 \%$ of the specimens) was found; however, that study included cT1-2, cN+ tumors whereas our analysis was limited to cT3 cancers with a negative mrCRM.

In conclusion, the results presented here suggest that omission of neoadjuvant chemoradiation prior to TME in mrCRM-negative, cT3 rectal cancer located at $<12 \mathrm{~cm}$ from the anal verge does not compromise local tumor control. However, caution is required when appraising these results because of the limited number of evaluable 
subjects (especially in the TME-alone group), which adds some uncertainty to the statistical analysis.

Such a policy should be limited to a setting where surgical expertise guarantees a high probability of obtaining a good-quality TME specimen, as well as to patients in whom a minimum distance between the circumferential tumor margin and the intended plane of resection of $>1$ $\mathrm{mm}$ is seen on pretherapeutic MRI. Obviously, adequate MRI imaging is essential for pursuing this concept.

As only limited data regarding the long-term outcome following TME alone exist from the era when good-quality TME was established in routine care, further studies are needed before a general recommendation to omit neoadjuvant radiation in any subset of rectal cancer patients can be given.

\section{Statement of Ethics}

This study was performed according to the guidelines of the Declaration of Helsinki for Biomedical Research from 1964 by the World Medical Association and its further amendments, the policy of the local institutional ethics committees, and the requirements of good clinical research and good clinical practice.

Data generation, documentation, and evaluation was performed according to prerequisites of the data protection law of the German district Saxony-Anhalt and according to federal law.

With regard to the study concept, it can be stated that a (potential) danger for study participants can be definitely excluded.

This paper is exempt from ethical committee approval.

Only patient-associated data were registered independently of the (specific interests of single) patients.

There is no imponderable risk or side effect for the patient as may become possible with the use of any medication.

Furthermore, the register with patient-associated data was led according to the requirements of the German "Landes- und Bundesdatenschutzgesetz"; data were evaluated anonymously without any possible inference to an individual patient.
In addition, data was monitored, validated, and evaluated at an institution close to a university hospital and a tertiary center and with an associated University Medical School; one of their basic tasks is (according to the "Hochschulmedizingesetz" in Germany) to perform clinical research in addition to clinical care for patients.

Last but not least, leading such a register of patient data can be considered closed to epidemiological studies, which do not need any statement of an ethics committee, as well as according to the "Allgemeinen Vertragsbedingungen" of the local contract between the physician and the patient ("Arzt-Patienten-Vertrag") in its current version from 2006 , article $(\$) 16$, paragraph (subparagraph) 4 , according to which it is not permitted to evaluate anonymous data.

\section{Conflict of Interest Statement}

H.P., B.G., F.M., and I.G. have no conflict of interests to declare.

\section{Funding Sources}

The authors declare that there was no funding of any research aspects relevant to the presented study.

\section{Author Contributions}

H.P. was involved in the conception and design of this work and in data acquisition, analyzed and interpreted the data, and was responsible for drafting this work and revising it critically according the coauthors' additions and critics. F.M. was responsible for analysis and interpretation of the study data as well as for revising the draft. I.G. assisted in interpretation of data for this work and in revising this paper critically. B.G. developed the conception and design of this work; in addition, the senior author was part of data acquisition and analyzed and interpreted data. Furthermore, B.G. was responsible for drafting this work and revising it critically according to all of the details suggested by the coauthors. All of the authors gave final approval of this work for publication.

\section{References}

1 Påhlman L, Glimelius B. Local recurrences after surgical treatment for rectal carcinoma. Acta Chir Scand. 1984;150(4):331-5.

2 Hermanek P, Mansmann U, Altendorf-Hofmann A, Hermanek P Jr, Riedl S, Staimmer D. [Comparative study of oncological outcome quality in colorectal carcinoma-ranking by surrogate endpoint?]. Chirurg. 1999 Apr; 70(4):407-14

3 Kapiteijn E, Marijnen CA, Colenbrander AC, Klein Kranenbarg E, Steup WH, van Krieken $\mathrm{JH}$, et al. Local recurrence in patients with rectal cancer diagnosed between 1988 and 1992: a population-based study in the west Netherlands. Eur J Surg Oncol. 1998 Dec;24(6):528-35.

4 Cedermark B, Johansson H, Rutqvist LE, Wilking N; Stockholm Colorectal Cancer Study Group. The Stockholm I trial of preoperative short term radiotherapy in operable rectal carcinoma. A prospective randomized trial. Cancer. 1995 May;75(9):2269-75.
5 Swedish Rectal Cancer Trial. Local recurrence rate in a randomised multicentre trial of preoperative radiotherapy compared with operation alone in resectable rectal carcinoma. Eur J Surg. 1996 May;162(5):397-402.

6 Martling A, Holm T, Johansson H, Rutqvist LE, Cedermark B; Stockholm Colorectal Cancer Study Group. The Stockholm II trial on preoperative radiotherapy in rectal carcinoma: long-term follow-up of a populationbased study. Cancer. 2001 Aug;92(4):896-902.

7 Påhlman L, Glimelius B. Pre- or postoperative radiotherapy in rectal and rectosigmoid carcinoma. Report from a randomized multicenter trial. Ann Surg. 1990 Feb;211(2):187-95.

8 Heald RJ, Moran BJ, Ryall RD, Sexton R, MacFarlane JK. Rectal cancer: the Basingstoke experience of total mesorectal excision, 19781997. Arch Surg. 1998 Aug;133(8):894-9.

9 Martling AL, Holm T, Rutqvist LE, Moran BJ, Heald RJ, Cedemark B. Effect of a surgical training programme on outcome of rectal cancer in the County of Stockholm. Stockholm Colorectal Cancer Study Group, Basingstoke Bowel Cancer Research Project. Lancet. 2000 Jul;356(9224):93-6.

10 Kapiteijn E, Kranenbarg EK, Steup WH, Taat CW, Rutten HJ, Wiggers T, et al. Total mesorectal excision (TME) with or without preoperative radiotherapy in the treatment of primary rectal cancer. Prospective randomised trial with standard operative and histopathological techniques. Dutch ColoRectal Cancer Group. Eur J Surg. 1999 May; 165(5):410-20.

11 Peeters KC, Marijnen CA, Nagtegaal ID, Kranenbarg EK, Putter H, Wiggers T, et al.; Dutch Colorectal Cancer Group. The TME trial after a median follow-up of 6 years: increased local control but no survival benefit in irradiated patients with resectable rectal carcinoma. Ann Surg. 2007 Nov;246(5):693-701. 
12 van Gijn W, Marijnen CA, Nagtegaal ID, Kranenbarg EM, Putter H, Wiggers T, et al.; Dutch Colorectal Cancer Group. Preoperative radiotherapy combined with total mesorectal excision for resectable rectal cancer: 12 year follow-up of the multicentre, randomised controlled TME trial. Lancet Oncol. 2011 Jun;12(6):575-82.

13 Nagtegaal ID, van de Velde CJ, van der Worp E, Kapiteijn E, Quirke P, van Krieken JH; Cooperative Clinical Investigators of the Dutch Colorectal Cancer Group. Macroscopic evaluation of rectal cancer resection specimen: clinical significance of the pathologist in quality control. JClin Oncol.2002 Apr;20(7):1729_ 34.

14 Nagtegaal ID, van de Velde CJ, Marijnen CA, van Krieken JH, Quirke P; Dutch Colorectal Cancer Group; Pathology Review Committee. Low rectal cancer: a call for a change of approach in abdominoperineal resection. J Clin Oncol. 2005 Dec;23(36):9257-64.

15 Ptok H, Mundt A, Lippert H, Gastinger I. [Rectal cancer surgery in Germany - a 10-year-analysis based on the data of the "Institute of Quality Assurance in Operative Medicine" at the Otto-von-Guericke University Magdeburg]. Zentralbl Chir. 2013 Aug;138(4):418-26.

16 Gilbert A, Ziegler L, Martland M, Davidson S, Efficace F, Sebag-Montefiore D, et al. Systematic review of radiation therapy toxicity reporting in randomized controlled trials of rectal cancer: a comparison of patient-reported outcomes and clinician toxicity reporting. Int J Radiat Oncol Biol Phys. 2015 Jul;92(3): 555-67.

17 Kulu Y, Tarantino I, Billeter AT, Diener MK, Schmidt T, Büchler MW, et al. Comparative outcomes of neoadjuvant treatment prior to total mesorectal excision and total mesorectal excision alone in selected stage II/III low and mid rectal cancer. Ann Surg Oncol. 2016 Jan;23(1):106-13.

18 Fan WH, Wang FL, Lu ZH, Pan ZZ, Li LR, Gao YH, et al. Surgery with versus without preoperative concurrent chemoradiotherapy for mid/low rectal cancer: an interim analysis of a prospective, randomized trial. Chin J Cancer. 2015 Jun;34(9):394-403.

19 Frasson M, Garcia-Granero E, Roda D, FlorLorente B, Roselló S, Esclapez P, et al. Preoperative chemoradiation may not always be needed for patients with $\mathrm{T} 3$ and $\mathrm{T} 2 \mathrm{~N}+$ rectal cancer. Cancer. 2011 Jul;117(14):3118-25.

20 Nagtegaal ID, Quirke P. What is the role for the circumferential margin in the modern treatment of rectal cancer? J Clin Oncol. 2008 Jan;26(2):303-12.

21 Ptok H, Ruppert R, Stassburg J, Maurer CA, Oberholzer K, Junginger T, et al. Pretherapeutic MRI for decision-making regarding selective neoadjuvant radiochemotherapy for rectal carcinoma: interim analysis of a multicentric prospective observational study. J Magn Reson Imaging. 2013 May;37(5):1122-8.
22 Kreis ME, Ruppert R, Ptok H, Strassburg J, Brosi P, Lewin A, et al.; OCUM study group. Use of preoperative magnetic resonance imaging to select patients with rectal cancer for neoadjuvant chemoradiation-interim analysis of the German OCUM Trial (NCT01325649). J Gastrointest Surg. 2016 Jan;20(1):25-32.

23 Ptok H, Gastinger I, Lippert H. [Local diagnostics for rectal cancer. What is realistic?]. Chirurg. 2012 May;83(5):448-51.

24 Schmiegel W, Pox C, Adler G, Fleig W, Fölsch UR, Frühmorgen P, et al.; Deutsche Gesellschaft für Verdauungs- und Stoffwechselkrankheiten. [S3-Guidelines Conference "Colorectal Carcinoma" 2004]. Z Gastroenterol. 2004 Oct;42(10):1129-77.

25 Quirke P, Steele R, Monson J, Grieve R, Khanna S, Couture J, et al.; MRC CR07/NCIC-CTG CO16 Trial Investigators; NCRI Colorectal Cancer Study Group. Effect of the plane of surgery achieved on local recurrence in patients with operable rectal cancer: a prospective study using data from the MRC CR07 and NCIC-CTG CO16 randomised clinical trial. Lancet. 2009 Mar;373(9666):821-8.

26 Sebag-Montefiore D, Stephens RJ, Steele R, Monson J, Grieve R, Khanna S, et al. Preoperative radiotherapy versus selective postoperative chemoradiotherapy in patients with rectal cancer (MRC CR07 and NCIC-CTG C016): a multicentre, randomised trial. Lancet. 2009 Mar;373(9666):811-20. 